\title{
UNIVERSITY OF LUND RADIOCARBON DATES VI
}

Sören Håkansson

\author{
Radiocarbon Dating Laboratory, Department of Quaternary Geology \\ University of Lund, Sweden
}

\section{INTRODUCTION}

Most of the $\mathrm{C}^{14}$ measurements reported here were made between October 1971 and October 1972. Equipment, measurement, and treatment of samples are the same as reported previously (R., 1968, v. 10, p. 36-37; 1970 , v. 12, p. 534).

Age calculations are based on a contemporary value equal to 0.950 of the activity of NBS oxalic acid standard and on the "conventional" halflife for $\mathrm{C}^{14}$ of $5568 \mathrm{yr}$. Results are reported in years before 1950 (years B.P.), and in the A.D./B.c. scale. Errors quoted $( \pm 1 \sigma)$ include standard deviations of count rates for the unknown sample, contemporary standard, and background. Corrections for deviations from the "normal" $\mathrm{C}^{13} / \mathrm{C}^{12}$ ratio for terrestrial plants $\left(\delta \mathrm{C}^{13}=-25.0 \%\right.$ in the PDB scale) are applied for all samples; also for marine shells, because apparent age of recent marine shells is not always just counterbalanced by the effect of isotopic fractionation (cf. Recent marine shells series, this list). $\delta \mathrm{C}^{13}$ values quoted are relative to the PDB standard.

The remark, "undersized; diluted", in Comments means the sample did not produce enough $\mathrm{CO}_{2}$ to fill the counter to normal pressure and "dead" $\mathrm{CO}_{2}$ from anthracite was introduced to make up the pressure. "\% sample" indicates amount of $\mathrm{CO}_{2}$ derived from the sample present in the diluted counting gas; the rest is "dead" $\mathrm{CO}_{2}$. Organic carbon content reported for bone sample is calculated from yield of $\mathrm{CO}_{2}$ by combustion of pretreated collagen. Organic carbon lost during treatment is not included in calculated percentage.

The description of each sample is based on information provided by the submitter.

\section{ACKNOWLEDGMENTS}

The author thanks Kerstin Lundahl for sample preparations and routine operation of the dating equipment, and $\mathrm{R}$. Ryhage and his staff at the mass-spectrometric laboratory of Karolinska Inst., Stockholm, for the $\mathrm{C}^{13}$ analyses. Special thanks are due Christian Cavallin for assistance with the electronic equipment.

\section{SAMPLE DESGRIPTIONS}

I. GEOLOGIC SAMPLES

\section{A. Sweden}

\section{Lilla Toften series}

Sediment from ancient lake Lilla Toften, Västmanland, Central Sweden (59 $48^{\prime} \mathrm{N}$ Lat, 16 ${ }^{\circ} 17^{\prime} \mathrm{E}$ Long). Coll. 1970 and subm. by S. Welinder, Dept. Quaternary Geol., Univ. Lund. Depths given are below surface. Only weak pretreatment with HCl due to small samples. All 
samples diluted. Amount of $\mathrm{CO}_{2}$ from sample is given in Comments below as "\% sample".

\section{Lu-417. Lilla Toften 1, 1.025 to $1.075 m$}

$$
\begin{aligned}
& \mathbf{4 4 0} \pm \mathbf{6 0} \\
& \text { A.D. } 1510 \\
& \delta C^{13}=-27.3 \%
\end{aligned}
$$

Clayey mud. Picea increase. Comment: $46 \%$ sample. (3 1-day counts.)

\section{Lu-418. Lilla Toften 2, 1.075 to $1.125 m$}

$$
680 \pm 60
$$

$$
\text { A.D. } 1270
$$

$\delta C^{13}=-27.6 \%$

Clayey mud. Picea increase. Comment: $52 \%$ sample. (3 1-day counts.)

Lu-419. Lilla Toften 3, 1.475 to $1.525 m$

$2450 \pm 85$ 500 в.C.

$$
\delta C^{13}=-29.7 \% \text { o }
$$
counts.)

Clayey mud. Rational Picea limit. Comment: 36\% sample. (3 1-day

\section{Lu-420. Lilla Toften 4, 1.525 to $1.575 m$}

$$
2660 \pm 80
$$
counts.)

Clayey mud. Rational Picea limit. Comment: $42 \%$ sample. (3 1-day

\section{Lu-421. Lilla Toften $5,2.575$ to $2.625 \mathrm{~m}$}

$5530 \pm 110$

3580 B.C.

$$
\delta C^{13}=-28.2 \%
$$
counts.)

Clayey mud. Quercus increase. Comment: $42 \%$ sample. (3 1-day

\section{Lu-422. Lilla Toften 6, 2.625 to $2.675 \mathrm{~m}$}

$$
5740 \pm 95
$$

3790 B.C.

$\delta C^{13}=-28.5 \%$ counts.)

Clayey mud. Quercus increase. Comment: $50 \%$ sample. (3 1-day

\section{Lu-423. Lilla Toften 7, 3.550 to $3.625 \mathrm{~m}$}

$6400 \pm 120$

4450 в.C.

$\delta C^{13}=-29.4 \%$ o

Clayey brackish mud. Rational Tilia limit. Comment: $53 \%$ sample.

\section{Lu-424. Lilla Toften 8, 3.625 to $3.700 \mathrm{~m}$}

$6510 \pm 100$

4560 в.c.

$\delta C^{13}=-28.8 \%$ c

Clayey brackish mud. Rational Tilia limit. Comment: $40 \%$ sample. (3 1-day counts.)

$$
\begin{array}{ll}
\text { Lu-534. Lilla Toften } 9+10,1.825 \text { to } 1.925 m & \begin{array}{r}
3910 \pm 70 \\
1960 \text { B.c. } \\
\delta C^{13}=-29.4 \% 0
\end{array}
\end{array}
$$

Clayey mud. Ulmus decrease. Comment: $77 \%$ sample. 


\section{Myrby series}

Sediment from small ancient lake at the farm Myrby, Västmanland, Central Sweden (59 $42^{\prime} \mathrm{N}$ Lat, $16^{\circ} 31^{\prime}$ E Long). Coll. 1971 and subm. by S. Welinder. Depths given are below surface. Pretreated with HCl only.

Lu-536. Myrby $1,1.30$ to $1.35 m$

Clayey mud. Beginning of Plantago.

\section{Lu-537. Myrby 2, 1.35 to $1.40 m$}

Clayey mud. Beginning of Plantago.

\section{Lu-538. Myrby 3, 1.60 to $1.65 \mathrm{~m}$}

$4160 \pm 60$ 2210 B.C. $\delta C^{13}=-27.3 \%$ $4080 \pm 65$ 2130 B.C. $\delta C^{13}=-26.3 \%$

$4930 \pm 85$ 2980 B.C. $\delta C^{13}=-24.1 \%$

Clayey brackish mud. Supposed 1st traces of agriculture. Comment: sample undersized; diluted; $72 \%$ sample.

\section{Lu-539. Myrby 4, 1.65 to $1.70 \mathrm{~m}$}

$4870 \pm 75$ 2920 B.C. $\delta C^{13}=-24.1 \%$

Clayey brackish mud. Supposed 1st traces of agriculture. Comment: sample undersized; diluted; $76 \%$ sample.

\section{Frövisjön series}

Sediment from ancient lake Frövisjön, Västmanland, Central Sweden (59 42' 40" N Lat, 16 ${ }^{\circ} 29^{\prime} 40^{\prime \prime}$ E Long). Coll. 1971 and subm. by S. Welinder. Depths given are below surface. Pretreated with HCl only.

\section{Lu-615. Frövisjön $1+2,0.375$ to $0.475 m$}

Mud. Quercus increase.

\section{Lu-616. Frövisjön $3+4,0.775$ to $0.875 m$}

Clayey mud. Rational Picea limit.

\section{Lu-617. Frövisjön $5+6,0.975$ to $1.075 m$}

Clayey mud. Empiric Picea limit.

Lu-618. Frövisjön 7, 2.325 to $2.425 m$

Clayey brackish mud.

$$
1940 \pm 55
$$$$
\text { A.D. } 10
$$$$
\delta C^{13}=-25.1 \% \text { o }
$$

$$
\mathbf{2 7 0 0} \pm \mathbf{5 5}
$$

750 B.C.

$\delta C^{13}=-23.2 \%$ o

$$
2880 \pm 55
$$

930 в.c. $\delta C^{13}=-25.4 \%$

$4500 \pm 75$

2550 B.C.

$\delta C^{13}=-26.0 \%$ 


\section{Barsebäcksmossen series}

Sediment and peat from Barsebäcksmossen, $15 \mathrm{~km} \mathrm{~N}$ of city of Malmö, S Sweden (55 $46^{\prime} \mathrm{N}$ Lat, $12^{\circ} 58^{\prime} \mathrm{E}$ Long). Alt. 1 to $1.5 \mathrm{~m}$, area $1.5 \mathrm{sq} . \mathrm{km}$. Coll. 1968 to 1969 and subm. by G. Digerfeldt, Dept. Quaternary Geol., Univ. Lund. Barsebäcksmossen is an ancient Littorina lagoon. Dating is part of study of transgressions and regressions indicated by stratigraphy and diatom analysis. Samples come from reconnaissance profile from shallow part of ancient lagoon (Digerfeldt, 1972), and from main profile, central and deepest part of basin. Depths refer to bog surface. Pretreated with HCl only (except Lu-578, -641, and -642). Some samples were given prolonged acid treatment owing to presence of mollusk shells and other carbonates in abundance. One sample contained enough Cardium shells for separate dating. From 2 samples total $\mathrm{CaCO}_{3}$ fraction was separated for dating.

Barsebäcksmossen, reconnaissance profile:

\section{Lu-426. Barsebäcksmossen, 199 to $204 \mathrm{~cm}$}

$$
\begin{array}{r}
9640 \pm 95 \\
7690 \text { B.C. } \\
\delta C^{13}=-25.8 \%
\end{array}
$$

Cladium-magnocaricetum peat, moderately humified, underlying Pollen Zone Boundary PB/BO 1.

\section{Lu-427. Barsebäcksmossen, 175 to $180 \mathrm{~cm}$}

$8910 \pm 90$

6960 B.C.

$$
\delta C^{13}=-25.8 \%
$$

Cladium-magnocaricetum peat, moderately humified, in BO 1.

\section{Lu-428. Barsebäcksmossen, 160 to $165 \mathrm{~cm}$}

$8530 \pm 90$

6580 B.C.

$\delta C^{13}=-26.4 \%$

Cladium-magnocaricetum peat, moderately humified, at $\mathrm{BO} 1 / \mathrm{BO} 2$.

Lu-429. Barsebäcksmossen, 120 to $125 \mathrm{~cm}$

Brackish gyttja, in A'T 2.

Lu-430. Barsebäcksmossen, 100 to $105 \mathrm{~cm}$

Brackish gyttja, in AT 2.

\section{Lu-431. Barsebäcksmossen, 70 to $75 \mathrm{~cm}$}

Brackish gyttja, overlying AT 2/SB 1.

Lu-447. Barsebäcksmossen, 55 to $60 \mathrm{~cm}$

Brackish gyttja rich in mollusk shells, in SB 1 .
$6430 \pm 75$

4480 B.C.

$\delta C^{13}=-19.7 \%$

$5980 \pm 70$

4030 B.c.

$\delta C^{13}=-18.2 \%$

$5470 \pm 65$

3520 B.C.

$\delta C^{13}=-18.5 \%$

$5210 \pm 65$

3260 B.C.

$\delta C^{13}=-19.7 \%$ 


\section{Lu-449. Barsebäcksmossen, 40 to $45 \mathrm{~cm}$}

Brackish gyttja rich in mollusk shells, in SB 1.

\section{Lu-578. Barsebäcksmossen, 30 to $35 \mathrm{~cm}$}

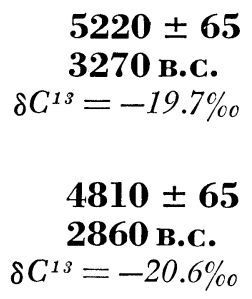

$5220 \pm 65$

3270 в.C.

$\delta C^{13}=-19.7 \%$

$4810 \pm 65$

2860 B.C.

$\delta C^{13}=-20.6 \%$ o

Brackish gyttja, in SB 1. Comment: because of proximity to surface and abundant root hairs, sample was specially treated for removal of root hairs and humus. Treated material was divided in coarse and fine fraction by sieving ( $2 \mathrm{~mm}$ net), and dated separately. Both fractions yielded same age $(4810 \pm 65)$.

\section{Lu-432. Barsebäcksmossen, 120 to $125 \mathrm{~cm}$, Cardium \\ $6540 \pm 140$ 4590 в.C. \\ $\delta C^{13}=+1.5 \%$}

Cardium shells separated from Lu-429. Comment: sample undersized; diluted; $42 \%$ sample.

$$
\begin{aligned}
& \text { Lu-448. Barse } \quad 5100 \pm 90 \\
& 3150 \text { B.c. } \\
& \delta C^{13}=-0.3 \%
\end{aligned}
$$

Total $\mathrm{CaCO}_{3}$ fraction separated from Lu-447. Comment: sample undersized; diluted; $48 \%$ sample. (3 1-day counts.)

$$
\begin{gathered}
\mathbf{3 0 4 0} \text { B.c. } \\
\delta C^{13}=-0.3 \% o
\end{gathered}
$$

Total $\mathrm{CaCO}_{3}$ fraction separated from Lu-449. Comment: sample undersized; diluted; $57 \%$ sample.

General Comment: corrections for deviations from "normal" $\mathrm{C}^{13} / \mathrm{C}^{12}$ ratio for terrestrial plants ( $\delta C^{1 s}=-25.0 \%$ in PDB scale) are applied also for shell and $\mathrm{CaCO}_{3}$ samples. No corrections are made for apparent age of shells of living marine mollusks (cf. Lu-234-236, R., 1969, v. 11, p. 441 and Lu-237, 1970, v. 12, p. 543). Preliminary results of study were pub. by submitter (Digerfeldt, 1972).

Barsebäcksmossen, main profile:

$$
\begin{aligned}
& 11,400 \pm 130 \\
& 9450 \text { в.C. } \\
& \delta C^{13}=-25.4 \%
\end{aligned}
$$

Lacustrine clay gyttja. Comment: undersized; diluted; $82 \%$ sample.

$$
\begin{array}{ll}
\text { Lu-694. Barsebäcksmossen, } 1037.5 \text { to } 1042.5 \mathrm{~cm} & \begin{array}{c}
9910 \pm 100 \\
7960 \text { B.c. } \\
\delta C^{13}=-31.0 \%
\end{array} \\
\text { Lacustrine clayey gyttja. } &
\end{array}
$$


$\begin{array}{cc} & \mathbf{8 6 0} \pm 110 \\ \text { Lu-636. Barsebäcksmossen, } 1025 \text { to } 1030 \mathrm{~cm} & \begin{array}{c}7010 \mathrm{B.C} . \\ \delta C^{13}=-30.2 \% \circ\end{array}\end{array}$

Lacustrine clayey gyttja. Comment: undersized; diluted; $78 \%$ sample.

Lu-637. Barsebäcksmossen, 990 to $995 \mathrm{~cm}$

Lacustrine gyttja.

Lu-570. Barsebäcksmossen, 962.5 to $967.5 \mathrm{~cm}$

Lacustrine gyttja.

Lu-569. Barsebäcksmossen, 950 to $955 \mathrm{~cm}$

Probably brackish gyttja.

Lu-689. Barsebäcksmossen, 835 to $840 \mathrm{~cm}$ Brackish gyttja.

Lu-690. Barsebäcksmossen, 735 to $740 \mathrm{~cm}$

Brackish gyttja.

Lu-638. Barsebäcksmossen, 625 to $630 \mathrm{~cm}$ Brackish gyttja.

Lu-639. Barsebäcksmossen, 560 to $565 \mathrm{~cm}$ Brackish gyttja.

Lu-691. Barsebäcksmossen, 510.5 to $515.0 \mathrm{~cm}$ Brackish gyttja.

Lu-640. Barsebäcksmossen, 455 to $460 \mathrm{~cm}$ Brackish gyttja.

Lu-561. Barsebäcksmossen, 345 to $350 \mathrm{~cm}$ Brackish gyttja. 
Lu-560. Barsebäcksmossen, 257.5 to $262.5 \mathrm{~cm}$

Brackish gyttja.

Lu-692. Barsebäcksmossen, 135 to $140 \mathrm{~cm}$

Lacustrine gyttja.

Lu-641. Barsebäcksmossen, 55 to $60 \mathrm{~cm}$
$4010 \pm 65$

2060 B.C.

$\delta C^{13}=-23.8 \%$

$3150 \pm 55$

1200 B.c.

$\delta C^{13}=-28.0 \%$

$2280 \pm 55$

330 в.c.

$\delta C^{13}=-28.0 \%$

Lacustrine gyttja. Comment: pretreated with $\mathrm{HCl}$ and $\mathrm{NaOH}$.

\section{Lu-641 A. Barsebäcksmossen, 55 to $60 \mathrm{~cm}$} humic acid

$2250 \pm 60$

300 B.C.

Acid-precipitated part of $\mathrm{NaOH}-$-soluble fraction from $\mathrm{Lu}-641$.

$$
\delta C^{13}=-27.4 \% \text { o }
$$

Lu-642. Barsebäcksmossen, 37.5 to $42.5 \mathrm{~cm}$

$1160 \pm 50$

A.D. 790

Carr peat, highly humified.

Lu-642 A. Barsebäcksmossen, 37.5 to $42.5 \mathrm{~cm}$, humic acid

Acid-precipitated part of $\mathrm{NaOH}$-soluble fraction from $\mathrm{Lu}-642$. Comment: undersized; diluted; $82 \%$ sample.

\section{Lu-592. Sandön}

$$
\begin{aligned}
& \quad \mathbf{1 0 8 0} \pm \mathbf{5 0} \\
& \text { A.D. 870 } \\
& \delta C^{13}=-25.1 \%
\end{aligned}
$$

Wood from outer part of oak stump from $2.5 \mathrm{~m}$ depth in Lake Vättern, near Sandön I., ca. $10 \mathrm{~km} \mathrm{NW}$ of Motala $\left(58^{\circ} 33^{\prime} \mathrm{N}\right.$ Lat, $14^{\circ} 52^{\prime} \mathrm{E}$ Long). Coll. 1969 by Göran Lord; subm. by G. Digerfeldt. Pretreated with $\mathrm{HCl}$ and $\mathrm{NaOH}$.

\section{Vassijaure series}

Sediment (Livingstone core sampler, diam. 36mm) from small lake, ca. $150 \times 150 \mathrm{~m}, 1100 \mathrm{~m}$ ENE of p. 514.22 near Vassijaure, Torne Lappmark, N Sweden $\left(68^{\circ} 25^{\prime} \mathrm{N}\right.$ Lat, $18^{\circ} 17^{\prime} \mathrm{E}$ Long). Alt. $480 \mathrm{~m}$, close to timberline in sub-alpine region. Coll. 1966 and subm. by M. Sonesson, Dept. Plant Ecol., Univ. Lund. Dating is part of study on vegetational dynamics and peat development in Torneträsk area (Sonesson, 1968; 1970) a, b). Other dates in series were reported previously (R., 1969, v. 11, p. 441-443). Depths given in sample titles refer to lake water level. Water depth $3.0 \mathrm{~m}$ at sampling point. Pretreated with HC1 only. All samples undersized; diluted. Amount of $\mathrm{CO}_{2}$ from sample is given in Comments below as "\% sample". 


\title{
Lu-503. Vassijaure, Gp 4, 395 to $405 \mathrm{~cm}$
}

Detritus gyttja and clay, at Zone Boundary T1/T2. Comment (M.S.): date agrees well with previous dates for same boundary (cf. R., 1969, v. 11, p. 442). $40 \%$ sample.

\section{Lu-622. Vassijaure, Gp 4, 365 to $375 \mathrm{~cm}$}

$5940 \pm 115$

3990 B.C.

$\delta C^{13}=-29.5 \%$

Detritus gyttja, at and underlying probable Zone Boundary T2/T3. Comment (M.S.): date much younger than corresponding boundary in Abisko valley (cf. R., 1969, v. 11, p. 442-443). 50\% sample.

\section{Lu-623. Vassijaure, Gp 4, 355 to $365 \mathrm{~cm}$ \\ $5520 \pm 130$ \\ 3570 B.c. \\ $\delta C^{13}=-29.2 \%$}

Detritus gyttja, overlying probable Zone Boundary T2/T3. Comment (M.S.): same as for Lu-622. 33\% sample. (3 1-day counts.)

\section{Lu-624. Vassijaure, Gp 4, 330 to $340 \mathrm{~cm}$}

$4570 \pm 125$

2620 B.C.

$\delta C^{13}=-27.9 \%$

Detritus gyttja, at supposed Zone Boundary T3/T4. Comment (M.S.): date too old for that boundary. $38 \%$ sample.

\section{Lu-625. Vassijaure, Gp 4, 316 to $325 \mathrm{~cm}$}

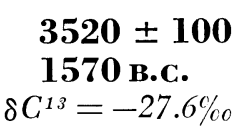

Detritus gyttja, at supposed Sub-Zone Boundary T4a/T4b. Comment (M.S.): date instead indicates Zone Boundary T3/T4. 42\% sample.

\begin{abstract}
Abisko series
Sediment (Livingstone core sampler, diam. 36mm) from sub-alpine lakes in Abisko valley, Torne Lappmark, N Sweden. Coll. 1963 and subm. by M. Sonesson. Dating is part of study on vegetational dynamics and peat development in Torneträsk area (Sonesson, 1968; 1970a, b). Other dates from Abisko valley were reported previously (R., 1969, v. 11, p. 441-443). Depths given refer to lake water level. Pretreated with $\mathrm{HCl}$ only. All samples except Lu-579 and -582 were undersized; diluted. Amount of $\mathrm{CO}^{2}$ from sample is given in Comments below as "\% sample".

\section{Lu-627. Vuolep Njakajaure, Gp 3, 1530 to $1540 \mathrm{~cm}$}
$7910 \pm 115$
5960 в.c.
$\delta C^{13}=-30.7 \%$

Detritus gyttja, underlying Sub-Zone Boundary T2a/b, Lake Vuolep Njakajaure $\left(68^{\circ} 20^{\prime} \mathrm{N}\right.$ Lat, $18^{\circ} 48^{\prime} \mathrm{E}$ Long). Alt. $409 \mathrm{~m}$; size ca. $500 \times 600 \mathrm{~m}$. Water depth $12.5 \mathrm{~m}$ at sampling point. Comment: $64 \%$ sample.
\end{abstract}


Lu-628. Vuolep Njakajaure, Gp 3,

$7460 \pm 90$ 1520 to $1530 \mathrm{~cm}$

Detritus gyttja, overlying Sub-Zone Boundary T2a/b. Comment: $88 \%$ sample.

Lu-629. Vuolep Njakajaure, Gp 3,

$4270 \pm 90$ 1415 to $1425 \mathrm{~cm}$ 2320 B.C. $\delta C^{13}=-30.6 \%$

Detritus gyttja, underlying Sub-Zone Boundary T3a/b. Comment: $57 \%$ sample.

Lu-630. Vuolep Njakajaure, Gp 3, 1405 to $1415 \mathrm{~cm}$

$$
\begin{array}{r}
\mathbf{4 0 4 0} \pm 90 \\
2090 \text { B.C. } \\
\delta C^{13}=-30.6 \%
\end{array}
$$

Detritus gyttja, underlying Sub-Zone Boundary T3a/b. Comment: $57 \%$ sample.

Lu-631. Vuolep Njakajaure, Gp 3, 1290 to $1300 \mathrm{~cm}$

Detritus gyttja, overlying Sub-Zone Boundary T4b/c. Comment: $36 \%$ sample.

\section{$10,680 \pm 120$ \\ Lu-580. Tjåutjanjarka N, F1:4, 360.5 to $366.5 \mathrm{~cm} \quad 8730$ в.c. \\ $\delta C^{13}=-21.4 \%$}

Sample from transition zone clay/detritus gyttja from N-most lake on cape Tjåutjanjarka at mouth of Abisko valley $\left(68^{\circ} 22^{\prime} \mathrm{N}\right.$ Lat, $18^{\circ} 56^{\prime} \mathrm{E}$ Long). Alt. $370 \mathrm{~m}$; size ca. $300 \times 400 \mathrm{~m}$. Water depth $1.6 \mathrm{~m}$ at sampling point. Comment: $82 \%$ sample.

\section{$10,820 \pm 110$ \\ Lu-579. Tjåutjanjarka $S$, E1 :6, 1047 to $1052 \mathrm{~cm} 8870$ в.c. $\delta C^{13}=-26.8^{c} \%$}

Sample from transition zone clay/detritus gyttja from lake ca. $500 \mathrm{~m}$ $\mathrm{SE}$ of sampling place for Lu-580. Alt. $383 \mathrm{~m}$; size ca. $300 \mathrm{x} 400 \mathrm{~m}$. Water depth $6.9 \mathrm{~m}$ at sampling point.

\section{Lu-581. Kartinvare, D2:3, 580 to $585 \mathrm{~cm}$}

$$
\begin{gathered}
\mathbf{9 8 4 0} \pm \mathbf{1 3 0} \\
\mathbf{7 8 9 0} \text { B.c. } \\
\delta C^{13}=-25.4 \% 0
\end{gathered}
$$

Sample from transition zone clay/detritus gyttja from lake $2.2 \mathrm{~km} \mathrm{NW}$ of p. 1154 (Mt. Kartinvare), ca. $17 \mathrm{~km} \mathrm{SW}$ of mouth of Abisko valley (68 ${ }^{\circ}$ $15^{\prime} \mathrm{N}$ Lat, $18^{\circ} 33^{\prime} \mathrm{E}$ Long). Alt. $51 \mathrm{~lm}$; size ca. $150 \times 300 \mathrm{~m}$. Water depth $3.8 \mathrm{~m}$ at sampling point. Comment: $71 \%$ sample. 


\section{Lu-582. Pajeb Njakajaure, P3 :6, 529 to $534 \mathrm{~cm} \quad 8640$ в.C. \\ $\delta C^{13}=-28.3 \%$}

Sample from transition zone clay/detritus gyttja from Lake Pajeb Njakajaure $\left(68^{\circ} 20^{\prime} \mathrm{N}\right.$ Lat, $18^{\circ} 50^{\prime}$ E Long). Alt. $469 \mathrm{~m}$; size ca. $200 \mathrm{x} 400 \mathrm{~m}$. Water depth $3.1 \mathrm{~m}$ at sampling point.

\section{Luopakte series}

Peat from perennially frozen mire, size ca. $50 \times 100 \mathrm{~m}$, on low-alpine part of Mt. Luovare, $1.2 \mathrm{~km}$ SSE of p. 627.89, Torne Lappmark, N Sweden (68 $14^{\prime}$ N Lat, 19²9' E Long). Alt. 670m. Coll. 1963 and subm. by M. Sonesson. Dating is part of study on vegetational dynamics and peat development in Torneträsk area (Sonesson, 1968; 1970a, b). For other dates from this site, see R., 1971, v. 13, p. 343. Depths given refer to surface of mire.

\section{Lu-632. Luopakte, Bp 140, 85 to $95 \mathrm{~cm}$}

$6520 \pm 110$

4570 B.C.

$\delta C^{13}=-27.1 \%$

Highly humified peat, at supposed Boundary T2/T3. Comment (M.S.): date confirms supposition. No pretreatment due to small sample; diluted; $57 \%$ sample.

\section{Lu-633. Luopakte, Bp 140, 70 to $80 \mathrm{~cm}$}

$6160 \pm 130$

4210 в.C.

$\delta C^{13}=-28.7 \%$

Highly humified peat, at boundary between minerogenous and ombrogenous peat (composed $i$. a. of Dicranum cf. elongatum). Comment: no pretreatment due to small sample; diluted; $18 \%$ sample. (2 2-day counts.)

\section{Lu-634. Luopakte, Bp 140, 40 to $50 \mathrm{~cm}$}

$4180 \pm 100$

2230 B.C.

$\delta C^{13}=-27.4 \%$

Relatively highly humified peat, at and below Boundary T3/T4. Comment (M.S.): date plausible considering low rate of peat deposition and Lu-635. Only weak HC1 pretreatment due to small sample; diluted; $50 \%$ sample.

\section{Lu-635. Luopakte, Bp 140, 30 to $40 \mathrm{~cm}$}
$3070 \pm 75$
1120 B.C.
$\delta C^{13}=-27.1 \%$

Relatively highly humified peat, at and above Boundary T3/T4. Comment (M.S.): see Lu-634. Only weak HC1 pretreatment due to small sample; diluted; $48 \%$ sample. (3 1-day counts.)

\section{Bysjön series}

Sediment from Lake Bysjön; near coast of central Blekinge $\left(56^{\circ} 11^{\prime}\right.$ N Lat, $15^{\circ} 08^{\prime}$ E Long). Site and samples of main sec. Bysjön I (R., 1971, 
v. 13, p. 344-345) and Bysjön III (R., 1972, v. 14, p. 382-383) described earlier. New series is derived from Bysjön II, a sec. in lake near shore studied to determine the sub-recent sedimentation rate. Water depth $140 \mathrm{~cm}$. Depths given are below lake water level. Coll. 1971 and subm. by B. E. Berglund, Dept. Quat. Geol., Univ. Lund. Pretreated with HCl only.

\section{Lu-614. Bysjön 19, 220 to $230 \mathrm{~cm}$}

$420 \pm 55$

A.D. 1530

$\delta C^{13}=-30.4^{\%} \%$

Lacustrine detritus gyttja; end of Pollen Zone SA 2. Comment: undersized; diluted; $77 \%$ sample.

\section{Lu-621. Bysjön 20, 190 to $200 \mathrm{~cm}$}

Lacustrine detritus gyttja. Comment: sample activity corresponds to radiocarbon age $10 \pm 50$ B.P.

General Comment (B.E.B.): analyses indicate unusually rapid sedimentation in this part of lake.

\section{Lu-620. Skillinge 4}

$6150 \pm 70$

4200 B.C.

$\delta C^{13}=-24.0 \%$

Peat from lowest of 5 layers of allochthonous organic material in sand from Littorina stage of the Baltic Sea, $0.9 \mathrm{~m}$ below surface on building site No. 258, 550m NNE of Skillinge harbor, E Scania $\left(55^{\circ} 29^{\prime} \mathrm{N}\right.$ Lat, $14^{\circ} 17^{\prime}$ E Long). Complement to earlier dates from Skillinge (R., 1970, v. 12, p. 545). Coll. 1969 by W. Vortisch; subm. by B. E. Berglund. Pretreated with $\mathrm{HCl}$ only.

\section{B. Poland}

\section{Lu-613. Lake Pakoskie, peat}

Peat from $1.4 \mathrm{~m}$ depth in lake terrace at Lake Pakoskie, ca. $45 \mathrm{~km} \mathrm{SW}$ of Torun, Voivodeship Bydgoszcz, N Poland $\left(52^{\circ} 43^{\prime} \mathrm{N}\right.$ Lat, $18^{\circ} 06^{\prime} \mathrm{E}$ Long). Terrace plane 3 to $4 \mathrm{~m}$ above present lake water level. Peat $0.2 \mathrm{~m}$ thick, overlain by $0.7 \mathrm{~m}$ sandy silt and lake marl with shells and plant fossils, underlain by $0.4 \mathrm{~m}$ marly sand with gravel and intercalations of peat. Coll. 1972 and subm. by W. Niewiarowski, Dept. Geog., Univ. Torun, Poland. Part of study of Holocene lake water level fluctuation in $\mathrm{N}$ Poland. Pretreated with $\mathrm{HCl}$ and $\mathrm{NaOH}$.

Lu-613A. Lake Pakoskie, humic acid

$$
\begin{gathered}
3480 \pm 60 \\
1530 \text { B.c. } \\
\delta C^{13}=-27.2 \% 0
\end{gathered}
$$

Acid-precipitated part of $\mathrm{NaOH}-$-soluble fraction from $\mathrm{Lu}-613$. 
East Greenland series (II)

$$
\text { C. Greenland }
$$

Marine shells from emerged marine sediments in Kong Oscars FjordVega Sund dist., NE Greenland. Coll. 1971 and subm. by C. Hjort, Dept. Quaternary Geol., Univ. Lund; part of 3-yr program for study of ice oscillations and shoreline displacement. For other dates from area, see R., 1972, v. 14, p. $388-390$.

$$
\begin{aligned}
& +2600 \\
& 38,400 \\
& \delta C^{13}=+0.1 \% \text { o }
\end{aligned}
$$

\section{Lu-532. Kap Mackenzie, 120 to $126 \mathrm{~m}$}

Shell fragments (Hiatella arctica and Mya truncata) from silty clay at +120 to $126 \mathrm{~m}$ on $\mathrm{mt}$. constituting Kap Mackenzie $\left(72^{\circ} 54^{\prime} \mathrm{N}\right.$ Lat, $21^{\circ} 54^{\prime}$ W Long), Geographical Society ö. Clay deposited in exposed position but undisturbed by ice; thus, probably postdates last ice-advance (Funder and Hjort, ms. in preparation). Comment: outer $22 \%$ of shells removed by acid leaching. (4 1-day counts.)

\section{Lu-533. Kap Mackenzie, 53m}

$$
\begin{gathered}
9610 \pm 95 \\
7660 \text { в.c. } \\
\delta C^{13}=+0.4 \% c
\end{gathered}
$$

Shells (Hiatella arctica and Mya truncata) from upper bed, $+53 \mathrm{~m}$, in marine stratigraphic sequence and now lying on abrasion terrace at this alt., a few $\mathrm{km}$ inland from Kap Mackenzie $\left(72^{\circ} 54^{\prime} \mathrm{N}\right.$ Lat, $21^{\circ} 58^{\prime} \mathrm{W}$ Long). Comment: outer $40 \%$ of shells removed by acid leaching.

\section{Lu-584. Gåseö, Bed D}

$5100 \pm 75$

3150 B.C.

$$
\delta C^{13}=-17.9 \%
$$

Organic material (mainly algae) from shell-bearing sandy sediment, Bed D, in transgressive sequence (Hjort, 1973a), Gåseö (72 48' N Lat, $22^{\circ} 55^{\prime}$ W Long), Scott Kelties Öer, Vega Sund. Comment: pretreated with HC1 only. Sample undersized; diluted; $58 \%$ sample. (3 1-day counts.)

\section{Lu-585. Gåsë̈, Bed C}

$6190 \pm 70$

4240 B.C.

$$
\delta C^{13}=+1.7 \%
$$

Shells (Hiatella arctica and Mya truncata) from sand, Bed C, in same sequence as Lu-584. Comment: outer $22 \%$ of shells removed by acid leaching.

\section{Lu-586. Gåseö, Bed E}

$$
\begin{gathered}
6470 \pm 70 \\
4520 \text { в.C. } \\
\delta C^{13}=+1.7 \%
\end{gathered}
$$

Shells (Hiatella arctica and Mya truncata) from silty clay, Bed $\mathrm{E}$, in same sequence as Lu-584 and -585. Comment: outer $26 \%$ of shells removed by acid leaching. 


\section{Lu-607. Adam af Bremens Dal}

Shell fragments (Hiatella arctica and Mya truncata) from an upper bed in stratigraphic sequence reaching $+60 \mathrm{~m}$, Adam af Bremens Dal $\left(72^{\circ}\right.$ $49^{\prime} \mathrm{N}$ Lat, $22^{\circ} 35^{\prime}$ W Long), Geographical Society Ö. Shells from +40 to $47 \mathrm{~m}$ (Funder and Hjort, ms. in preparation). Comment: outer $22 \%$ of shells removed by acid leaching. (3 1-day counts.)

\section{Lu-608. Kap Elisabeth}

$$
\begin{aligned}
& 8090 \pm 80 \\
& 6140 \text { B.c. } \\
& \delta C^{13}= \pm 0 \% o
\end{aligned}
$$

Shells (Hiatella arctica, Mya truncata, and Mytilus edulis) from abrasion terrace in silty clay at $+52 \mathrm{~m}$, Kap Elisabeth $\left(72^{\circ} 55^{\prime} \mathrm{N}\right.$ Lat, $24^{\circ} 48^{\prime}$ W Long), Ella Ö. Highest and oldest known occurrence of Mytilus in area (cf. Noe-Nygaard, 1932). Comment: outer $23 \%$ of shells removed by acid leaching.

\section{Lu-645. Snaevringen, inner fraction}

$9660 \pm 95$ 7710 B.c.

$$
\delta C^{13}=+0.6 \%
$$

Shells (Mya truncata) from abrasion terrace in silty clay at $+55 \mathrm{~m}$, Snaevringen $\left(72^{\circ} 44^{\prime} \mathrm{N}\right.$ Lat, $23^{\circ} 00^{\prime} \mathrm{W}$ Long), Vega Sund (Traill ö). Comment: inner fraction $(51 \%$ of shells) was used.

\section{Lu-644. Snaevringen, outer fraction}

$9540 \pm 105$ 7590 B.C.

$\delta C^{13}=+0.6 \%$

Outer fraction of shells used for Lu-645. Comment: outer fraction corresponds to $41 \%$ of shells; outermost $8 \%$ removed by acid leaching. Sample undersized; diluted; $85 \%$ sample.

\section{Lu-646. Kap Laura}

$9740 \pm 90$

7790 B.C

$\delta C^{13}=-0.6 \%$

Shells (Mya truncata) from sandy silty bed in delta built up to $+65 \mathrm{~m}$, Kap Laura ( $72^{\circ} 53^{\prime} \mathrm{N}$ Lat, $23^{\circ} 25^{\prime} \mathrm{W}$ Long), Vega Sund (Geog. Soc. Ö). Shells from $57 \mathrm{~m}$. Comment: outer $9 \%$ of shells removed by acid leaching.

\section{Lu-647. Segldal}

$9610 \pm 95$

7660 B.C.

$\delta C^{1.3}=+1.4 \%$

Shells (Hiatella arctica) from silty clay covering outermost of terminal moraines in valley mouth, Segldal $\left(72^{\circ} 07^{\prime} \mathrm{N}\right.$ Lat, $23^{\circ} 34^{\prime} \mathrm{W}$ Long), Scoresby Land (Kong Oscars Fjord). Shells from $+57 \mathrm{~m}$. Comment: outer $18 \%$ of shells removed by acid leaching. 


\section{Lu-648. Kirschdalen}

Shells (Hiatella arctica and Mya truncata) from silty clay, Kirschdalen (72 $34^{\prime}$ N Lat, $24^{\circ} 42^{\prime}$ W Long), Lyells Land (Kong Oscars Fjord). Shells from $+50 \mathrm{~m}$ but clay is found to $55 \mathrm{~m}$. Shells probably date marked shoreline at $65 \mathrm{~m}$. Comment: outer $40 \%$ of shells removed by acid leaching.

\section{Lu-649. Oxedalen}

$8660 \pm 85$

6710 B.c.

$\delta C^{13}=+0.2 \%$

Shells (Hiatella arctica and Mya truncata) from sandy silty beds in delta built up to $+40 \mathrm{~m}$, Oxedal $\left(72^{\circ} 06^{\prime} \mathrm{N}\right.$ Lat, $23^{\circ} 50^{\prime} \mathrm{W}$ Long), Scoresby Land (Mesters Vig). Comment: outer $35 \%$ of shells removed by acid leaching. General Comment: corrections for deviations from "normal" $\mathrm{C}^{13} / \mathrm{C}^{12}$ ratio for terrestrial plants $\left(\delta C^{13}=-25.0 \%\right.$ in PDB scale) are applied also for shell samples. No corrections are made for apparent age of shells of living marine mollusks. For apparent age in area, cf. Recent marine shells series, below, and paper by submitter (Hjort, 1973b).

\section{Recent marine shells series}

Shells of marine mollusks coll. alive during Swedish expedition to NE Greenland in 1899 and 1900. Samples were stored in alcohol at Swedish Mus. Nat. Hist., Stockholm. Selected and subm. by C. Hjort.

\section{Lu-609. Mackenziebugt, inner fraction}
Apparent age: $600 \pm 47$ $\delta C^{13}=-0.3 \%$ o

Shells (Hiatella arctica, Modiolaria laevigata, Clinocardium [Cardium] ciliatum, and Serripes [Cardium] groenlandicus) from 12 to $35 \mathrm{~m}$ depth in Mackenziebugt, NE Greenland ( $73^{\circ} 28^{\prime} \mathrm{N}$ Lat, $21^{\circ} 30^{\prime} \mathrm{W}$ Long). Coll. 1900 by Swedish Zool. Polar Expedition. Comment: inner fraction (55\% of shells) was used.

\section{Lu-610. Mackenziebugt, outer fraction}

$$
\begin{array}{r}
\text { Apparent age: } \mathbf{5 7 0} \pm \mathbf{5 4} \\
\delta C^{13}=+0.2 \%
\end{array}
$$

Outer fraction of shells used for Lu-609. Comment: outer fraction corresponds to $35 \%$ of shells; outermost $10 \%$ removed by acid leaching. Sample undersized; diluted; $73 \%$ sample.

\section{Lu-643. Fame öer}

$$
\begin{array}{r}
\text { Apparent age: } 590 \pm \mathbf{3 9} \\
\delta C^{13}=+0.1 \%
\end{array}
$$

Shells (Tridonta [Astarte] borealis) from 5 to $8 \mathrm{~m}$ depth at Fame öer, Scoresby Sund $\left(70^{\circ} 50^{\prime} \mathrm{N}\right.$ Lat, $22^{\circ} 33^{\prime} \mathrm{W}$ Long). Coll. 1899 by Swedish NE Greenland Expedition. Comment: outer $15 \%$ removed by acid leaching. Sample undersized; diluted; $89 \%$ sample. (3 1-day counts.) 
Lu-650. Pendulumöer/Claveringöen Apparent age: $540 \pm 38$ $\delta C^{13}=+1.3 \%$

Shells (Tridonta $[$ Astarte $]$ borealis and Tridonta [Astarte] elliptica) from 18 to $21 \mathrm{~m}$ depth S of L. Pendulumöen $\left(74^{\circ} 35^{\prime} \mathrm{N}\right.$ Lat, $18^{\circ} 23^{\prime} \mathrm{W}$ Long) and from 25 to $40 \mathrm{~m}$ depth SE of Claveringöen $\left(74^{\circ} 10^{\prime} \mathrm{N}\right.$ Lat, $20^{\circ}$ $08^{\prime}$ W Long). Coll. 1899 by Swedish NE Greenland Expedition. Comment: outer $14 \%$ removed by acid leaching. (3 1-day counts.)

General Comment: corrections for deviations from "normal" $\mathrm{C}^{13} / \mathrm{C}^{12}$ ratio for terrestrial plants $\left(\delta C^{13}=-25.0 \%\right.$ in PDB scale) are applied also for these shells. Age corrections are made for time between collection and 1950. Results are discussed by submitter (Hjort, 1973b).

\section{ARCHAEOLOGIC SAMPLES}

\section{Viken series}

\section{Sweden}

Wood from large part of oak tree from peat, $50 \mathrm{~cm}$ thick, ca. $150 \mathrm{~cm}$ below surface at $\mathrm{N}$. Hage, Viken, NW Scania, S Sweden $\left(56^{\circ} 09^{\prime} \mathrm{N}\right.$ Lat, $12^{\circ}$ 34' 41" E Long). Coll. 1969 and subm. by B. Hulthén, Hist. Mus., Univ. Lund. Shape and working of wooden object resemble cult figures from Iron age found in other parts of N Europe. Pretreated with HCl and $\mathrm{NaOH}$.

\section{Lu-571. Viken, Sample 1}

Wood from outer part of tree.

\section{Lu-619. Viken, Sample 2}

A.D. 1600

$\mathbf{3 5 0} \pm \mathbf{5 0}$

$$
\delta C^{13}=-25.3 \%
$$

$$
\text { A.D. } 1360
$$$$
\mathbf{5 9 0} \pm \mathbf{5 0}
$$$$
\delta C^{13}=-25.0 \%
$$

Wood cores taken radially from surface to middle of trunk.

General Comment (B.H.): dates younger than expected. Typologically, sample belongs from 100 B.c. to A.D. 100. Part of difference between dates probably due to secular variations in $\mathrm{C}^{14}$ activity (Suess, 1970). Bristle-cone pine-corrected historical date for Lu-571 is between A.D. 1420 and 1520 . No such correction for Lu-619.

\section{Skanör-Falsterbo series}

Wood from excavation of defense structure from Middle Ages at Ljungen, Skanör-Falsterbo, S Sweden ( $55^{\circ} 24^{\prime} \mathrm{N}$ Lat, $12^{\circ} 52^{\prime} \mathrm{E}$ Long). Coll. 1971 and subm. by G. Andersson, Hist. Mus., Univ. Lund. Pretreated with $\mathrm{HCl}$ and $\mathrm{NaOH}$.

\section{Lu-572. Ljungen, Sample 1}

Wood from outer part of oak post $30 \mathrm{~cm}$ thick assumed part of defense structure. Comment (G.A.): dated to determine age of entire construction. Date younger than expected. 


\section{Lu-573. Ljungen, Sample 3}

Wood from small branch in organic layer with seaweed on slope of hillock with defense structure. Comment (G.A.): dated to determine if hillock is natural or was formed during construction of defense structure.

\section{Maglarp series}

Human bones from excavation in old church at Maglarp, Scania $\left(55^{\circ}\right.$ $24^{\prime} \mathrm{N}$ Lat, $13^{\circ} 05^{\prime} \mathrm{E}$ Long). Church is one of the earliest brick churches in Sweden, possibly from end of 12th century. Coll. 1970 and subm. by B. Sundnér, Hist. Mus., Univ. Lund. Samples treated as described previously (R., 1970, v. 12, p. 534).

\section{Lu-575. Maglarp, Sample 1}

$$
980 \pm 50
$$

Collagen from well preserved human tibia from grave in $\mathrm{N}$ part of chancel. Alt. $+4.50 \mathrm{~m}$. Comment (B.S.): grave may belong to earlier wooden church on same place. Date is satisfactory. Organic carbon content: $6.2 \%$.

\section{Lu-576. Maglarp, Sample 2}

$$
\begin{aligned}
& \mathbf{9 1 0} \pm \mathbf{5 0} \\
& \text { A.D. } 1040 \\
& \delta C^{13}=-18.2 \%
\end{aligned}
$$

Collagen from well preserved human bones (calcanea and metatarsals) from brick-built grave covered with limestone slabs in $S$ part of tower. Alt. $+5.29 \mathrm{~m}$. Comments (B.S.): grave probably from earliest time of brick church, i.e., ca. A.D. 1150, but not earlier in view of grave construction. (S.H.): considering known variations in $\mathrm{C}^{14}$ activity (Suess, 1970), possible historic date is between A.D. 1030 and 1200. Organic carbon content: 7.1\%.

\section{Lu-603. Norrvidinge, House 339}

\section{A.D. 1820}

$130 \pm 50$

Wood fraoments from wall no longer standing of House 339 at Norrvidinge $3^{22-23}$, Scania, S Sweden $\left(55^{\circ} 51^{\prime} \mathrm{N}\right.$ Lat, $13^{\circ} 07^{\prime} \mathrm{E}$ Long). Site is rich in ceramics (Late Neolithic-Early Bronze age). Coll. 1971 and subm. by J. Callmer, Hist. Mus., Univ. Lund. Pretreated with $\mathrm{HCl}$ and $\mathrm{NaOH}$. Comment (J.C.): modern date indicates that sample, from upper part of fill, may be connected with disturbances in area by farming activities in middle of 19th century and later.

\section{Lu-604. Löddesborg, Grave 9}

$3760 \pm 65$

1810 B.c.

$\delta C^{13}=-25.7 \%$

Charcoal from remains of wooden construction in Grave 9 at Löddesborg, Löddeköpinge parish, Scania, S Sweden $\left(55^{\circ} 44^{\prime} \mathrm{N}\right.$ Lat, $13^{\circ} 00^{\prime} \mathrm{E}$ Long). Grave construction is unique, combining 2 very different grave 
types. Assoc. with flint artifacts (2 daggers, 2 spearheads, 1 borer, and 1 scraper). Coll. 1970 and subm. by J. Callmer. Pretreated with HCl and $\mathrm{NaOH}$. Comment (J.C.): date agrees fairly well with archaeol. dating of daggers in grave to later part of Late Neolithic.

\section{Hagestad series}

Charcoal and bone from Hagestad, Löderup parish, Scania $\left(55^{\circ} 23^{\prime}\right.$ N Lat, $14^{\circ} 09^{\prime}$ E Long). Coll. 1971 and subm. by M. Strömberg, Hist. Mus., Univ. Lund. For other dates from Hagestad, see R., 1972, v. 14, p. 394-395. Charcoal pretreated with $\mathrm{HCl}$ and $\mathrm{NaOH}$. Bone treated as described previously (R., 1970, v. 12, p. 534).

\section{Lu-652. Hagestad 6 ${ }^{2}$ A, Sample 1:HT 1971}

$$
\begin{aligned}
2030 & \pm 55 \\
\mathbf{8 0} \text { в.C. } & \\
\delta C^{13}=-21.2 \% & -2 \%
\end{aligned}
$$

Collagen from well preserved cattle bone (scapula) from field $S$ of brook, $\mathrm{N}$ of coast rd., Hagestad $6^{2} \mathrm{~A}$. Comment: organic carbon content: $4.9 \%$.

\section{Lu-653. Hagestad 6² A, Sample 2:HT 1971}

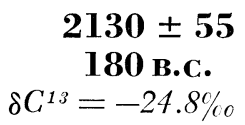

Charcoal from hearth in Trench A:1971 on same field as Lu-652.

\section{Lu-654. Hagestad 6² A, Sample 3:HT 1971}

$$
\begin{array}{r}
2060 \pm 55 \\
110 \text { в.c. } \\
\delta C^{13}=-23.6 \%
\end{array}
$$
-653 .

Charcoal from hearth in Trench 1:1971 on same field as Lu-652 and

\section{Lu-655. Hagestad 26, Sample 4:HT 1971}

$$
\begin{gathered}
\mathbf{1 9 3 0} \pm \mathbf{5 5} \\
\text { A.D. } 20 \\
\delta C^{13}=-23.9 \%
\end{gathered}
$$
stad $26^{6}$.

Charcoal from hearth in Trench 1:1971 on field $S$ of coast rd., Hage-

\section{Lu-656. Hagestad 26 ${ }^{6}$, Sample 5:HT 1971}

$$
\begin{gathered}
2070 \pm 55 \\
120 \text { в.c. } \\
\delta C^{13}=-23.2 \%
\end{gathered}
$$

Charcoal from hearth in Trench 2:1971 on same field as Lu-655. General Comment (M.S.): all dates agree well with archaeol. results based on assoc. finds.

\section{Löderup series}

Charcoal from grave field with different burial forms at Löderup 15, Löderup parish, Scania ( $55^{\circ} 23^{\prime} \mathrm{N}$ Lat, $14^{\circ} 07^{\prime} \mathrm{E}$ Long). Site is closely connected with investigated area at Hagestad. Coll. Nov. 1971 to Jan. 1972 and subm. by M. Strömberg. Pretreated with $\mathrm{HCl}$ and $\mathrm{NaOH}$. 


\section{Lu-657. Löderup 15, Grave 8:71}

Charcoal from bottom layer of Grave 8:71 (inhumation burial). Assoc. with many amber beads of unusual form.

\section{Lu-658. Löderup 15, Grave 35}

Charcoal from bed in Late Neolithic grave (inhumation burial).

Lu-659. Löderup 15, Grave 27

$3810 \pm 60$

1860 в.C.

Charcoal from bed in grave with burnt bones. Assoc. with fragment of bronze tweezers.

General Comment (M.S.): date Lu-658 agrees well with archaeol. results, but Lu-657 and -659 are older than expected (Iron-age features). Discrepancy may be explained by possibility that earlier wooden Stone-age constructions were burnt with re-use of grave field by Iron-age people.

\section{Gårdlösa series}

Charcoal from Gårdlösa, Smedstorp parish, SE Scania $\left(55^{\circ} 34^{\prime} \mathrm{N}\right.$ Lat, $14^{\circ} 08^{\prime}$ E Long). Coll. 1964 to 1970 and subm. by B. Stjernquist, Hist. Mus., Univ. Lund. Dated for study of continuity of Iron-age settlement in Gårdlösa area. For other dates from area and references, see R., 1972, v. 14, p. 264-266, 392-393.

\section{Lu-651. Gårdlösa 2, Ship-setting}

Charcoal from hearth close to stone of ship-setting. Depth ca. $60 \mathrm{~cm}$. Coll. 1970. Comment: pretreated with $\mathrm{HCl}$ and $\mathrm{NaOH}$.

\section{Lu-662. Gårdlösa 2, Grave 51}

$$
\begin{aligned}
& \mathbf{1 2 6 0} \pm \mathbf{5 0} \\
& \text { A.D. } 690 \\
& \delta C^{13}=-24.1 \%
\end{aligned}
$$

Charcoal from grave with burnt bones. Depth. ca. $50 \mathrm{~cm}$. Comment: pretreated with $\mathrm{HCl}$ and $\mathrm{NaOH}$.

\section{Lu-663. Gårdlösa 2, Grave Field $1967: 2$}

$$
\begin{gathered}
2050 \pm 60 \\
100 \text { B.c. } \\
\delta G^{13}=-24.7 \%
\end{gathered}
$$

Charcoal from concentration of burnt bones inside quadratic stone setting, depth 50 to $60 \mathrm{~cm}$, covered by eolian sand and humus layer. Assoc. with 2 glass beads. Coll 1967. Comment: no pretreatment due to small sample; diluted; $85 \%$ sample.

\section{Lu-664. Gårdlösa 2, Grave Field $1967: 18$}

$$
\begin{gathered}
1980 \pm 60 \\
\text { 30 B.C. } \\
\delta C^{13}=-24.1 \% \circ
\end{gathered}
$$

Charcoal from concentration of burnt bones below stone. Assoc. with 
rivet washer. Coll. 1967. Comment: no pretreatment due to small sample; diluted; $78 \%$ sample.

\section{Lu-665. Gårdlösa 2, Grave Field $1967: 23$}

$$
\begin{aligned}
& \quad \mathbf{1 5 1 0} \pm \mathbf{5 0} \\
& \text { A.D. } 440 \\
& \delta C^{13}=-23.5 \%
\end{aligned}
$$

Charcoal from hearth covered by eolian sand and humus, inside circular stone setting. Assoc. with burnt clay and undatable sherds of pottery. Coll. 1967. Comment: pretreated with $\mathrm{HCl}$ and $\mathrm{NaOH}$.

\section{Lu-666. Gårdlösa 2, Grave Field $1967: 26$}

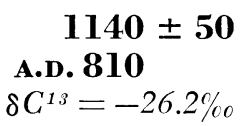

Charcoal from feature with brittle-burnt stones, soot, and burnt bones. Assoc. with part of bird-fibula, melted glass bead, and a piece of resin. Dept ca. $60 \mathrm{~cm}$. Coll. 1967. Comment: only $\mathrm{NaOH}$ pretreatment due to small sample.

\section{Lu-667. Gårdlösa 2, Grave Field $1967: 47$}

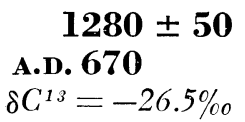

Charcoal from hearth connected with stones in circular stone setting. Depth ca. $60 \mathrm{~cm}$. Coll. 1967. Comment: pretreated with $\mathrm{HCl}$ and $\mathrm{NaOH}$.

\section{Lu-668. Gårdlösa 3, Hearth No. 40}

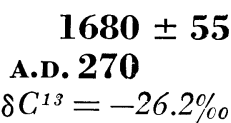

Charcoal from Hearth No. 40 connected with Spring III on cult-place (Stjernquist, 1964). Depth ca. $60 \mathrm{~cm}$. Coll. 1964. Comment: pretreated with $\mathrm{HCl}$ and $\mathrm{NaOH}$.

\section{Lu-669. Gårdlösa 3, Grave 70}

$$
\begin{aligned}
& 1680 \pm 60 \\
& \text { A.D. } 270 \\
& \delta C^{13}=-24.4 \%
\end{aligned}
$$

Charcoal from pit below triangular stone setting. Depth ca. $50 \mathrm{~cm}$. Coll. 1969. Comment: only weak HCI pretreatment due to small sample; diluted; $67 \%$ sample.

General Comment (B.S.): dates Lu-663 and -664 are 300 to 400 yr older than expected from archaeol. situation. All other dates agree well with estimates based on archaeol. material.

\section{Lu-670. Saxtorp 6:12 \\ $1280 \pm 50$}

Sample from wooden well-lining from excavation at Saxtorp $6: 12$ Saxtorp parish, Scania (55 $50^{\prime} 30^{\prime \prime} \mathrm{N}$ Lat, $12^{\circ} 58^{\prime} 10^{\prime \prime} \mathrm{E}$ Long). Well bottom ca. $150 \mathrm{~cm}$ below surface, covered by 4 differently colored sand layers. Coll. 1970 and subm. by J. Petersson, Royal Office Antiquities. Cellulose fraction separated and dated because of possible superficial contamination with polyglycol. Comment (J.P.): well cannot be dated accu- 
rately with archaeol. material. Only $0.5 \mathrm{~m}$ from well a hearth was found containing Late Bronze-age pottery, but radiocarbon date agrees with archaeol. age estimates for other features in excavation area.

\section{Önsvala series}

Charcoal from 3 out of 13 hearths spatially connected with Iron-age grave field dated A.D. 300 to 950 and Viking-age settlement, at önsvala 5:1, 1800m NE of Mölleberga church, Nevishög parish, Scania (55 $37^{\circ} 30^{\prime \prime}$ $\mathrm{N}$ Lat, $13^{\circ} 13^{\prime} 50^{\prime \prime}$ E Long). Coll. 1969 and subm. by J. Petersson, Hist. Mus., Univ. Lund. Pretreated with $\mathrm{HCl}$ and $\mathrm{NaOH}$.

\section{Lu-672. Önsvala 5:1, Feature 61}

Charcoal from hearth ca. $35 \mathrm{~cm}$ below surface.

\section{Lu-673. Önsvala 5:1, Feature 68}

Charcoal from hearth ca. $35 \mathrm{~cm}$ below surface.

\section{Lu-671. Önsvala 5:1, Feature 70}

Charcoal from hearth ca. $35 \mathrm{~cm}$ below surface.

$$
\begin{gathered}
\mathbf{1 . 6 0 0} \pm \mathbf{5 0} \\
\text { A.D. 350 } \\
\delta C^{13}=-24.4 \% 0
\end{gathered}
$$$$
\begin{aligned}
& 1290 \pm \mathbf{5 0} \\
& \text { A.d. } \mathbf{6 6 0} \\
& \delta C^{13}=-25.0 \%
\end{aligned}
$$$$
1590 \pm 50
$$$$
\text { A.D. } 360
$$$$
\delta C^{13}=-24.7 \%
$$

General Comment (J.P.): dates agree well with archaeol. dates of artifacts from oldest part of grave field.

\section{Löddeköpinge series}

Animal bones and teeth from excavation of Viking-age settlement at Löddeköpinge, E Scania (55 $45^{\prime} \mathrm{N}$ Lat, $13^{\circ} 00^{\prime} \mathrm{E}$ Long). Coll. 1965 to 1967 and subm. by T. Ohlsson, Hist. Mus., Univ. Lund. Teeth received same treatment as bone (R., 1970, v. 12, p. 534).

\section{Lu-684. Löddek̈̈pinge, Sample 1, bone}

$$
\begin{aligned}
& \mathbf{1 1 7 0} \pm \mathbf{5 0} \\
& \text { A.D. } 780 \\
& \delta C^{13}=-20.9 \%
\end{aligned}
$$

Collagen from poorly preserved animal bone fragments from House 1, Field A. Assoc. with Viking-age pottery. Coll. 1965. Comment: organic carbon content: $3.5 \%$.

\section{Lu-685. Löddeköpinge, Sample 1, teeth}

Collagen from well preserved animal teeth from same position as Lu-684. Coll. 1965. Comment: organic carbon content: $2.3 \%$.

\section{Lu-686. Löddeköpinge, Sample 2}

$$
\begin{aligned}
& \mathbf{1 1 3 0} \pm \mathbf{8 5} \\
& \text { A.D. 820 } \\
& \delta C^{13}=-21.4 \%
\end{aligned}
$$

Collagen from poorly preserved animal bone fragments from Feature 8, Field B. Assoc. with comb fragment and Viking-age pottery. Coll. 1966. 
Comment: organic carbon content: $0.7 \%$. Undersized; diluted; $33 \%$ sample. (3 1-day counts.)

\section{Lu-687. Löddeköpinge, Sample 3}

$1120 \pm 50$ A.D. 830

$\delta C^{13}=-21.7 \%$

Collagen from poorly preserved animal bone fragments from Feature 13, Field B. Assoc. with Viking-age pottery. Coll. 1966. Comment: organic carbon content: $2.5 \%$.

\section{Lu-688. Löddeköpinge, Sample 4}
$1120 \pm 50$
A.D. 830
$\delta C^{13}=-20.9 \%$

Collagen from fairly well preserved animal bone from Feature 11, Field G. Assoc. with iron objects and local as well as imported Viking-age pottery. Coll. 1967. Comment: organic carbon content: $1.8 \%$.

\section{Lu-699. Löddeköpinge, Sample 5}
$1130 \pm 50$ A.D. 820 $\delta C^{13}=-21.7 \%$ c

Collagen from well preserved animal teeth from Feature 10, Field K. Assoc. with oval brooch (early type), iron objects, and Viking-age pottery. Coll. 1967. Comment: organic carbon content: $1.8 \%$.

General Comment (T.O.): dates are made on bone fragments and teeth, i.e., refuse from cooking and butchering. Samples are chosen to date activities and are from different parts of settlement. Radiocarbon dates closely correspond to archaeol. dates based on analysis of artifacts from houses. Radiocarbon dates confirm that settlement was occupied during 1st half of Viking age (9th century).

\section{REFERENCES}

Digerfeldt, G., 1972, A preliminary report of an investigation of Littorina transgressions in the Barsebäck area, western Skane: Gcol. Fören. Stockholm Förh., v. 94, p. 537-548.

Hjort, C., 1973a, The Vega Transgression-a hypsithermal event in Central East Greenland: Geol. Soc. Denmark Bull., v. 22, p. 25-38. v. 95 , no. 1 .

Noe-Nygaard, A., 1932, Remarks on Mytilus edulis in raised beaches in East Greenland: Grönland Medd., v. 95, no. 2, 23 p.

Sonesson, Mats, 1968, Pollen zones at Abisko, Torne Lappmark, Sweden: Bot. Notiser (Lund), v. 121, p. 491-500.

1970a, Studies on mire vegetation in the Torneträsk area, northern Sweden. III. Communities of the poor mires: Op. Bot. A Soc. Bot. Lundensi, v. 26, 120 p. 1970b, Studies on mire vegetation in the Torneträsk area, northern Sweden. IV. Some habitat conditions of the poor mires: Bot. Notiser (Lund), v. 123, p. 67-111. Stjernquist, Berta, 1964, New light on spring-cults in Scandinavian prehistory: Archacology, v. 17, no. 3, p. 180-184.

Suess, H. E., 1970, Bristlecone-pine calibration of the radiocarbon time-scale 5200 B.C. to the present, in: Olsson, I. U. (ed.), Radiocarbon variations and absolute chronology, 12th Nobel symposium proc., Uppsala: Stockholm, Almqvist \& Wiksells Förlag, p. 303-311, pl. I. 